\title{
PROPUESTA TÉCNICA PARA LA OBTENCIÓN DE QUITINA A PARTIR DE CAPARAZO- NES DE CRUSTÁCEOS A NIVEL DE PLANTA PILOTO
}

\author{
Martha Benavente Silva \\ Maestra en Procesamiento de Alimentos \\ Docente Investigadora, FIQ, UNI \\ mbena_98@yahoo.com
}

\section{RESUMEN}

Con este proyecto se logró determinar las condiciones óptimas para procesar caparazón de camarón y cola de langostino para la obtención de quitina. Así también, se determinaron los principales equipos para el montaje de la planta piloto, la cual podría ser ubicada en el laboratorio de operaciones unitarias de la FIQ. Este estudio servirá de referencia para realizar proyectos de investigación que involucre la obtención de valiosos productos a partir de desechos.

\section{INTRODUCCIÓN}

Actualmente, la producción de crustáceos ocupa el segundo lugar entre los principales rubros de exportación del país. Sin embargo, también ha creado un serio problema ambiental, ya que se estima que esta industria genera grandes volúmenes de desechos, los cuales, en su mayoría, son depositados sin ningún tratamiento previo.

Por otro lado, este material puede ser aprovechado como materia prima para la obtención de productos de interés comercial y de investigación, como es la quitina y el quitosano, los cuales presentan numerosas aplicaciones en distintas áreas, principalmente en medicina, farmacia, medioambiente, cosméticos e industria alimenticia, etc. (Harish et al., 2007).

Además, se puede obtener D-glucosamina, utilizada en el tratamiento de osteoartritis, una serie de proteínas, muy importantes en la nutrición acuícola y la astaxantina, un pigmento usado en el cultivo de salmones y tilapias (Agullo et al., 2007).

Con la elaboración de este estudio se pretende sentar las bases para en un futuro, realizar el montaje de una planta piloto con la capacidad de procesar 50 kilos de caparazón de camarón o langostino por lote, para la obtención de quitina con un rendimiento del $90 \%$ en base seca.

\section{MATERIAL Y REACTIVOS}

Para esta investigación se trabajó con muestras de desecho de crustáceos, camarón y cabeza de langostino, los cuales fueron proporcionados por la empresa CAMANICA y Acuario Internacional, respectivamente. Los reactivos utilizados para la extracción de la quitina fueron: hidróxido de sodio $(\mathrm{NaOH})$, ácido clorhídrico $(\mathrm{HCl})$, e hipoclorito de sodio ( $\mathrm{NaClO}$ ). Los reactivos fueron preparados con agua blanda ( $\sin$ contenido de calcio y magnesio).

\section{Parte experimental}

\%Contenido de humedad. Se pesaron $10 \mathrm{~g}$ de materia prima y se introdujeron en un horno a $105^{\circ} \mathrm{C}$ por 4 horas. Luego, la muestra se enfrió y se pesó. El calculó se basó en la Ec. (1).

$\%$ Contenido de humedad $=\frac{\mathrm{m}_{1}-\mathrm{m}_{2}}{\mathrm{~m}_{1}} \times 100$

$$
\text { Ec. (1) }
$$

Optimización de reactivos. Para optimizar la cantidad de reactivos se varió el volumen en cada una de las etapas principales: desproteinización, desmineralización y blanqueo. La Tabla 1 presenta las condiciones utilizadas en cada experimento. En el caso de la cabeza de langostino se cambiaron las concentraciones de $\mathrm{HCl}$ (de $1.8 \mathrm{~N}$ a $3.6 \mathrm{~N}$ ) 
Volumen de reactivo (L) por kg de material $\mathrm{NaOH} 10 \%$

$\mathrm{HCl} 1.8 \mathrm{~N}$ $\mathrm{NaClO} 0.38 \%$
Caparazón de camarón

No. de variable

$\begin{array}{ccc}\text { I } & \text { II } & \text { III } \\ 5 & 4 & 3 \\ 4 & 3 & 2 \\ 4 & 3 & 8\end{array}$

Cabeza de langostino

No. de variable

II
4
3
3

$\begin{array}{ccc}\text { III } & \text { IV } & \text { V } \\ 3 & 4 & 4 \\ 2 & 3 & 4 \\ 8 & 12 & 12\end{array}$

Tabla 1. Condiciones experimentales para el procesamiento de desechos de crustáceos.

y $\mathrm{NaClO}$ (de $0.38 \%$ a $0.76 \%$ ) para garantizar el blanqueo total de la quitina y así, obtener un producto de mejor calidad.

Propuesta técnica de la Planta Piloto. Dentro de la evaluación se consideró: escalamiento de los reactivos; dimensionamiento de los equipos para procesar $50 \mathrm{~kg}$ de material diario; propuesta técnica de diseño de la planta piloto y su microlocalización; y una evaluación económica preliminar en base al consumo de reactivos.

\section{RESULTADOS Y DISCUSIÓN}

La Tabla 2 presenta los resultados obtenidos en la determinación del \% Contenido de Humedad para el caparazón de camarón y cabeza de langostino sin trituración y con trituración. En esta Tabla se puede observar que el contenido de humedad promedio para cada material es muy alto en relación al contenido en base seca.

Descripción

Caparazón de camarón

Cabeza de langostino sin trituración

Cabeza de langostino con trituración

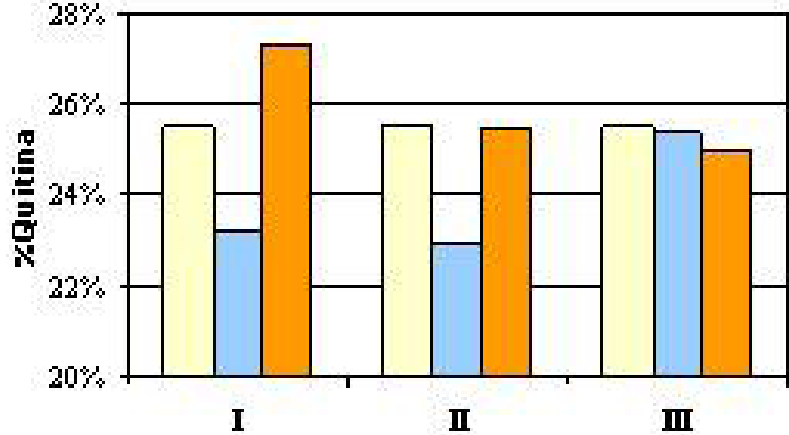

$\square$ Bibliografia* $\square$ Con trituración $\square$ Sin trituración

(a)

presentaba pigmentación y la textura del material era dura. Para obtener mejores resultados, para las variables IV y $\mathrm{V}$ se cambió la concentración del $\mathrm{HCl}$ a $3.6 \mathrm{~N}$ y del $\mathrm{NaClO}$ a $0.76 \%$, observándose que la apariencia del producto mejoró notablemente ya que éste era de color blanco y su textura era suave.

\%Contenido de humedad
76.70
70.40
68.00

\%Materia seca
23.30
29.60
32.00

Tabla 2. \%Contenido de humedad para los diferentes tipos de desechos de crustáceos.

La Figura 1 refleja los resultados experimentales de la producción de quitina. Estos resultados obtenidos muestran que los parámetros óptimos para procesar el caparazón de camarón fueron las condiciones experimentales de la variable III, ya que se obtuvo un producto blanco y de buena textura. Bajo estas condiciones de trabajo se obtuvo un \% Recuperación del 95\%. Para el caso de la cabeza de langostino se observó que con las condiciones experimentales de la variable I, II y III el producto
En base a la experiencia a nivel de laboratorio y los balances de masa se logró determinar y dimensionar los principales equipos: un tanque de reacción de 450L con agitador de hélice, tres tanques de $250 \mathrm{~L}$ para almacenamiento de reactivos, una bomba de agua y una balanza de $100 \mathrm{~kg}$. También se consideraron accesorios tales como tubos PVC, tubos $\mathrm{T}$, codos, válvulas, bridas, etc. 


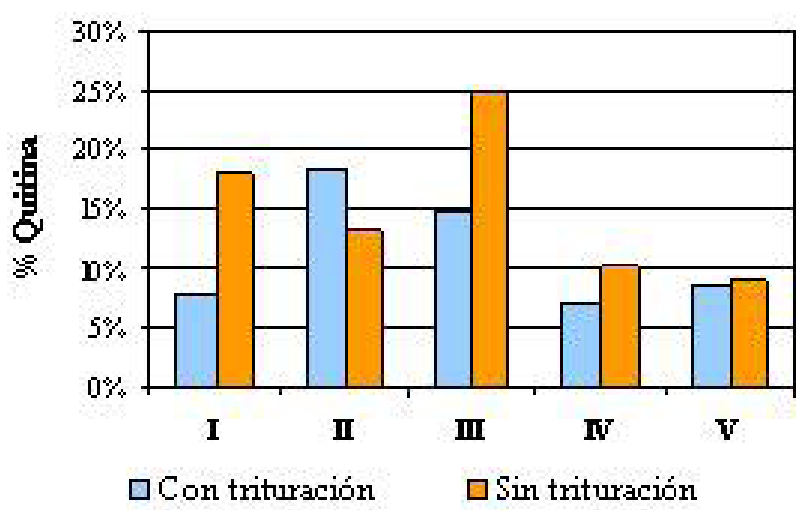

(b)

Figura 1. Resultados de \% quitina a partir de (a) caparazón de camarón y (b) cabeza de langostino

La evaluación económica, en base al consumo de reactivo, indicó que el costo para producir $1 \mathrm{~g}$ de quitosana a partir del caparazón de camarón fue de USD 0.03 y a partir de cabeza de langostino fue USD 0.12. Los cuales fueron altos en comparación con los precios internacionales.

\section{CONCLUSIÓN}

Las condiciones óptimas para procesar un $\mathrm{kg}$ de caparazón de camarón, fueron: $3 \mathrm{~L} \mathrm{NaOH}$ al $10 \%$, $2 \mathrm{~L} \mathrm{HCl} 1.8 \mathrm{~N}$ y $8 \mathrm{~L} \mathrm{NaClO}$ al $0.38 \%$. Mientras que para procesar un $\mathrm{kg}$ de cabeza de langostino, las condiciones óptimas fueron: $4 \mathrm{~L} / \mathrm{kg} \mathrm{NaOH}$ al $10 \%$, $4 \mathrm{~L} / \mathrm{kg}$ de $\mathrm{HCl} 3.6 \mathrm{~N}$ y $12 \mathrm{~L} / \mathrm{kg}$ de $\mathrm{NaOCl}$ al $0.76 \%$; sin trituración de la materia prima y con un tiempo de agitación de 2 horas en la etapa de desproteinización.

Bajo estas condiciones experimentales, se puede obtener un \% Recuperación mayor del 95\% en el caso del caparazón de camarón.

La evaluación económica indicó que el precio de un gramo de quitina producida tiene un costo muy alto, principalmente en el caso de la cabeza de langostino, en comparación con los precios internacionales.
Se debe reevaluar las condiciones de trabajo para disminuir los costos sin menoscabo de la calidad del producto.

\section{BIBLIOGRAFÍA}

- Agulló E., Mato R., Tapia C., Heras A., San Román J., Argüelles W., Goycoolea F., Mayorga A., Nakamatsu J., Pastor A., (2004). "Quitina y Quitosano: Obtención, Caracterización y Aplicaciones", Pontificia Universidad Católica del Perú, Fondo Editorial 2004, pp. 244 $-245$.

- Harish, K. V., y R. N. Tharanathan (2007). "Chitin/Chitosan Modifications and their unlimited Application Potencial - An Overiew". Trenes in Food Science \& Technology 18: 117 $-131$.

- Muzzarelli R. A. A., (1977). “Chitin”, Pergamon Press, New York.

\section{AGRADECIMIENTOS}

Los autores agradecen al Programa de Investigación y Desarrollo de la Vice-Rectoría de Investigación y Desarrollo, y al Programa UNI-ASDIFIQ por financiar este proyecto.

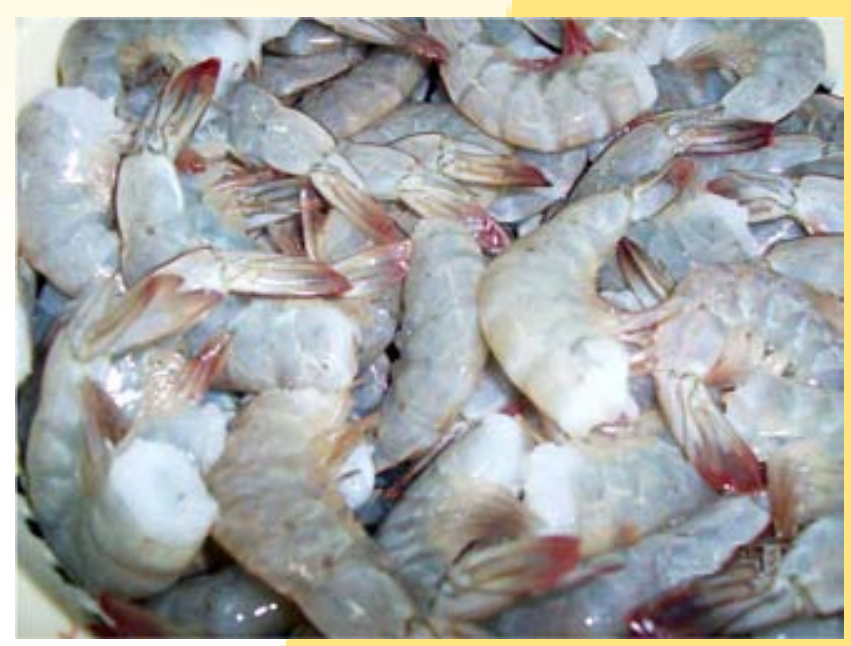

Materia prima para la quitina 